\title{
Comparison of Health Status in Primary Care Underserved Area Residents and the General Population in Korea
}

\author{
Min So Kim ${ }^{1}$, Kyae Hyung Kim ${ }^{1,2 *}$, Sang Min Park ${ }^{1,3}$, Jong-goo Lee ${ }^{1,3}$, Yeon Seo Ko ${ }^{1}$, A Ra Cho', Yoon Su Ku' \\ 'Department of Family Medicine, Seoul National University Hospital, Seoul, Korea \\ ${ }^{2}$ Institute for Public Health and Medical Service, Seoul National University Hospital, Seoul, Korea \\ ${ }^{3}$ Department of Family Medicine, Seoul National University College of Medicine, Seoul, Korea
}

Background: This study compared chronic diseases and health-related quality of life (HRQoL) in between primary care underserved areas residents and the general population.

Methods: Underserved areas were identified according to accessibility and the time relevance index for primary care. Overall, 279 participants aged $\geq 60$ years from four counties enrolled voluntarily. A total of 1,873 individuals were assigned in the control group using the Korea National Health and Nutrition Examination Survey database. We assessed the differences in prevalence, awareness, and control of hypertension and diabetes and HRQoL using both subjective health status and the Korean version of the EuroQol-5D (EQ-5D) questionnaire using multivariate logistic regression analysis between the two groups.

Results: For hypertension, prevalence did not differ significantly between the two groups, whereas awareness and control were lower in the underserved areas than that in the general population; the adjusted odds ratios (95\% confidence interval) were $0.40(0.25-0.64)$ and 0.27 (0.18-0.41), respectively. For diabetes, differences in prevalence, awareness, and control were statistically insignificant. The proportion reporting poor subjective health status and problems in four EQ-5D indexes (ability to exercise, daily activities, pain/discomfort, anxiety/depression) was higher in the underserved areas, which also had a lower EQ-5D index, than that in the general population.

Conclusion: Primary care underserved area residents were underdiagnosed and under-controlled for hypertension and reported poorer subjective health and HRQoL compared to the general population. Primary care is the attributable factor to awareness and control of chronic diseases and subjective health and QoL in communities.

Keywords: Medically Underserved Area; Health Status Disparities; Hypertension; Diabetes Mellitus; Quality of Life; Korea

Received: August 1, 2018, Revised: January 24, 2019, Accepted: January 28, 2019

${ }^{*}$ Corresponding Author: Kyae Hyung Kim https://orcid.org/0000-0001-9954-6422

Tel: +82-2-2072-2473, Fax: +82-2-2072-0374, E-mail: khkim@snuh.org 


\section{INTRODUCTION}

Chronic diseases have become the leading contributors to mortality and disease burden worldwide due to rapid aging and greater longevity. Care for chronically ill patients is characterized by underdiagnosis, undertreatment, and failure to use primary and secondary preventive measures. ${ }^{1)}$ It is also important to evaluate health-related quality of life (HRQoL) as health has become more than merely extending people's lifespan; maintaining a healthy lifestyle has been highlighted over the years. ${ }^{2)}$

Inequalities have been observed in health status based on area of residence. ${ }^{3-6)}$ Generally, hypertension and diabetes are significantly common in men, older adults, and unmarried people; those who are less educated and have lower incomes are also at higher risk than those who are highly educated and have higher incomes. ${ }^{1,7,8)}$ In particular, the prevalence of chronic disease has been reported to be higher in rural areas than in urban areas; residents of rural areas also have lower HRQoL than residents of urban areas. ${ }^{9,10)}$ Several studies on the prevalence, awareness, treatment, and control of hypertension and diabetes have shown different influences that can be attributed to rural residence. ${ }^{9,11-13)}$ Studies evaluating health status in medically underserved areas (MUAs) in Korea are insufficient. ${ }^{14)}$ Health status reported from the previous studies may differ because rural areas are not always MUAs.

An MUA is a "geographic location, which has insufficient health resources to meet the medical needs of the resident population."15) MUAs were defined using validated indexes of accessibility and the time relevance index (TRI) to identify places with poor access and quality of health care, respectively. ${ }^{14)}$ We conducted the present study in areas defined as MUA for primary care (primary care underserved area, PCUA). Primary care has promoted health and is associated with a more equitable distribution of health resources in populations compared with specialty care centers, specifically in rural areas. ${ }^{16)}$ We assessed health status by investigating the prevalence, awareness, and control of hypertension and diabetes in the underserved areas. We also evaluated HRQoL using self-reported questionnaires.

\section{METHODS}

\section{Data Sources and Participants}

We used two databases to evaluate the influence of area of residence defined as PCUAs. The databases used were the Korean rural health survey conducted by Seoul National University Hospital visiting 10 to 12 PCUAs annually and the Korea National Health and Nutrition Examination Survey (KNHANES).

The existence of Korean PCUAs was investigated by the Ministry of Health and Welfare in 2014. ${ }^{14)}$ Their analysis unit was the town, and they investigated areas using accessibility and TRI. Accessibility was defined as the percentage of the population who can reach primary care services within 30 minutes. Areas with less than $30 \%$ of accessibility were defined as underserved and regarded as having poor access to primary care. The TRI refers to the percentage of individuals among the total medical cases living within 30 minutes of a primary care clinic. Areas with less than $30 \%$ TRI were considered to have poor quality of primary care, which defines an underserved area. A previous study identified a total of 315 PCUAs. ${ }^{14)}$

We used the above mentioned Korean rural health survey, which was conducted by one national university hospital in 2017 . We collected information on the health status, residence area of the participants, and their clinical indexes. By sorting participants' areas of residence, we included four counties that were identified as PCUAs. The counties were Taean-gun (Chungcheongnam-do), Goseong-gun (Gyeongsangnam-do), Namwon-si (Jeonlabuk-do), and Yeongwol-gun (Gangwondo). A total of 306 participants, who were 60 years or older, from these four counties voluntarily enrolled in the study. All participants provided written informed consent for inclusion in the study.

The following data were assessed: measurement of blood pressure (BP) and serum blood glucose, self-reported clinical diagnosis of hypertension and diabetes, questionnaire of subjective health status (SHS), and the Korean version of the EuroQol-5D (EQ-5D). ${ }^{16)}$ For the control group, we used the data of the participants who were 60 years or older from the KNHANES 2016, a nationwide survey on the general health and nutritional status of the Korean population. ${ }^{17)}$ The survey used a stratified, multistage probability sampling design and identified 10,806 individuals from 13,289 households (4,416 households per year) and 576 districts (192 districts per year) based on location and residence to represent the entire nation. ${ }^{17)}$ We excluded participants who did not check their BP or blood glucose level, did not report a clinical diagnosis of hypertension and diabetes, and/or did not complete the SHS or EQ-5D questionnaire. After excluding these data, we compared the data from 1,873 KHNANES survey participants as the general population sample with those from the 279 residents of PCUAs (Figure 1).

\section{Outcome Variables and Co-variables}

According to standard protocols, systolic BP (SBP) and diastolic BP (DBP) were measured from the right arm 3 times, and the final BP measurement was obtained as the mean of the second and third results. We obtained glucose levels from a blood sample; we confirmed the appropriate fasting time. The "glucose level" variable was defined as glucose levels or random glucose levels based on an 8-hour fasting period.

"Hypertension" was defined as (1) a self-reported previous clinical diagnosis of hypertension and (2) measured SBP of $\geq 140 \mathrm{~mm} \mathrm{Hg}$ or DBP of $\geq 90 \mathrm{~mm}$ Hg. Among hypertensive participants, awareness of hypertension was defined as diagnosis by a doctor in a clinical setting. Controls were defined as those with SBP $<140 \mathrm{~mm} \mathrm{Hg}$ and DBP $<90$ $\mathrm{mm} \mathrm{Hg}$ among hypertensive participants.

"Diabetes" was defined as (1) a self-reported previous clinical diagnosis of diabetes and (2) glucose levels measuring above $126 \mathrm{mg} / \mathrm{dL}$ as serum fasting glucose level or above $200 \mathrm{mg} / \mathrm{dL}$ in a random glucose level measurement. Among patients with diabetes, "awareness of dia- 


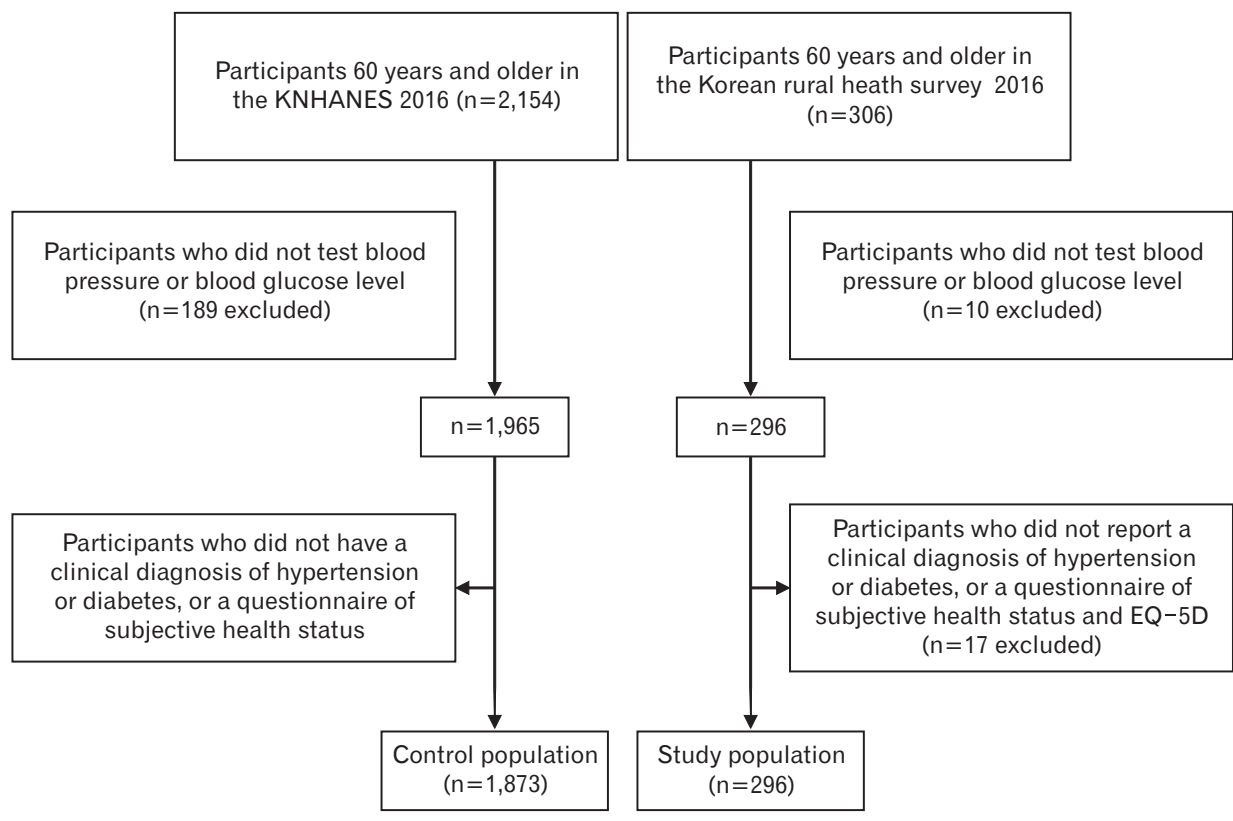

Figure 1. Flow diagram of the general population and the primary care underserved area participants. KNHANES, Korea National Health and Nutrition Examination Survey; EQ-5D, EuroQol-5D. betes" was defined as having been clinically diagnosed with diabetes by a doctor. "Control of diabetes" was defined when the measured serum level was below a fasting glucose of $130 \mathrm{mg} / \mathrm{dL}$ and random glucose of $180 \mathrm{mg} / \mathrm{dL}$ among patients with diabetes.

"Current smoking" was defined as currently smoking cigarettes regardless of the number of cigarettes, and "drinking" was defined as any alcohol intake of more than once per month during the last 12 months.

We used educational level, household income level, experience of being selected as basic living recipient by the government, and number of people living together as indicators of socioeconomic status (SES). Educational level was categorized as follows: lack of schooling or unschooled, elementary school graduate or below, junior or high school graduate or below, and college admission or above. Household income was defined as the average total monthly income (thousand won/mo) classified as less than 400, 400 to less than 900 , and 900 or more.

SHS was evaluated by checking the answer to the question "What do you think about your current health?" Participants selected one among the five possible answers of very bad, bad, usual, good, and very good. Although this questionnaire is more subjective than a clinical test, several previous studies have reported its association with mortality, chronic disease, and medical utility; thus, it has been extensively used to measure health status. ${ }^{18)}$ Survey respondents reporting their SHS as "bad" and "very bad" were more likely to show higher mortality than those reporting "very good" or "good" SHS. We categorized "very bad" and "bad" into the "bad participant health" group and "very good," "good," and "average" into the "good participant health" group. ${ }^{19)}$

The EQ-5D developed by the EuroQol Group is one of the most widely used tools for the assessment of HRQoL. We used the Korean version of the EQ-5D three-level version. ${ }^{16)}$ The Korean EQ-5D exhibit- ed good validity and sensitivity in the general population. ${ }^{16)}$ Participants responded "one among significant problems," "some problems," or "no problems" for each of the five indexes (ability to exercise, selfcare, daily activities, pain/discomfort, and anxiety/depression). We combined "some problem" and "significant problem" responses to form a "yes problem" response. ${ }^{19)}$ From the participants' answers, we obtained EQ-5D index score using the formula described below. The formula was calculated by the Korean Centers for Disease Control and Prevention as estimated weights of EQ-5D as follows: ${ }^{20)}$

$$
\begin{aligned}
\mathrm{Y}=1-(0.050+0.096 \times \mathrm{M} 2+0.418 \times \mathrm{M} 3+0.046 \times \mathrm{SC} 2+0.136 \times \mathrm{SC} 3+ & \\
& 0.051 \times \mathrm{UA} 2+0.208 \times \mathrm{UA} 3+0.037 \times \mathrm{PD} 2+0.151 \times \mathrm{PD} 3+0.043 \times \mathrm{AD} 2+ \\
& 0.158 \times \mathrm{AD} 3+0.050 \times \mathrm{N} 3)
\end{aligned}
$$

where M2=mobility level 2, M3=mobility level 3, SC2=self-care level 2, SC3=self-care level 3, UA2=usual activities level 2, UA3=usual activities level 3, PD2=pain or discomfort level 2, PD3=pain or discomfort level 3, AD2=anxiety or depression level 2, AD3=anxiety or depression level 3, N3=any dimension on level 3, level 1=no problem, level $2=$ some problem, and level $3=$ significant problem.

\section{Statistical Analyses}

We compared the general characteristics of the participants based on area of residence using t-tests and $\chi^{2}$ tests. We estimated the association between area of residence and hypertension and diabetes management using a multivariate logistic regression model. We adjusted for potential confounding factors, including age, sex, body mass index $\left(\mathrm{BMI}, \mathrm{kg} / \mathrm{m}^{2}\right.$ ), smoking status, drinking status, educational level, household income, and number of people living in the same household. We calculated the adjusted odds ratio (aOR) and 95\% confidence intervals (CIs). The HRQoL in physical health was evaluated using the 
SHS and Korean version of the EQ-5D questionnaires. We used a multivariate logistic regression model to assess the association between HRQoL and area of residence. The association between the index of EQ-5D and area of residence was evaluated using a multivariate linear regression model. All analyses were performed using Stata ver. 14.0 (Stata Corp., College Station, TX, USA). P-values $<0.05$ were considered statistically significant.

\section{RESULTS}

\section{Characteristics of the Study Population}

There were 1,873 participants from the general population and 279 from the PCUAs. The general characteristics of the study population by area of residence are shown in Table 1. Residents of PCUAs were older. However, no statistically significant difference in BMI or sex was observed between the two groups. PCUA residents showed better health behaviors (higher proportion of nonsmokers and nondrinkers) than the residents from the general population. There were significant dif- ferences in SES according to area of residence. Residents of PCUAs had lower educational levels and lower household income than the general population. Among the residents of PCUAs, $27.8 \%$ lived alone, compared with $19.91 \%$ of the general population.

\section{Prevalence, Awareness, and Control of Hypertension and Diabetes}

Prevalence, awareness, and control of hypertension and diabetes according to area of residence before adjustments are shown in Table 2. Hypertension was more prevalent in PCUAs than in the general population (70.61\% versus $60.79 \%, \mathrm{P}=0.002)$. Among hypertensive participants, awareness and control of their condition were significantly lower in PCUAs (84.71\% versus $72.59 \%$ and $61.07 \%$ versus $30.96 \%$, respectively; all $\mathrm{P}<0.001)$ than in the general population. The prevalence and awareness of diabetes was not significantly different in terms of area of residence ( $\mathrm{P}=0.360$ and $\mathrm{P}=0.306$, respectively). However, diabetic participants were more controlled in PCUAs (47.25\% versus $63.64 \%$, $\mathrm{P}=0.012$ ) than in the general population.

Table 1. Characteristics of the participants by area of residence: general population $(n=1,873)$ and Korean primary care underserved area $(n=279)$

\begin{tabular}{|c|c|c|c|}
\hline \multirow{2}{*}{ Characteristic } & \multicolumn{2}{|c|}{ Area of residence } & \multirow{2}{*}{ P-value } \\
\hline & General population $(n=1,873)$ & Primary care underserved area $(n=279)$ & \\
\hline Age (y) & $69.96(6.36)$ & $73.29(5.62)$ & $<0.001$ \\
\hline $60-69$ & $941(50.24)$ & $73(26.26)$ & $<0.001$ \\
\hline $70-79$ & $726(38.76)$ & $163(58.63)$ & \\
\hline$\geq 80$ & $206(11.00)$ & $42(15.11)$ & \\
\hline \multicolumn{4}{|l|}{ Sex } \\
\hline Female & $1,048(55.95)$ & $160(57.35)$ & 0.661 \\
\hline Male & $825(44.05)$ & $119(42.65)$ & \\
\hline Body mass index (kg/m²) & $24.29 \pm 3.2$ & $24.69 \pm 3.35$ & 0.058 \\
\hline Smoking & & & $<0.001$ \\
\hline Never & $1,140(61.46)$ & 245 (89.42) & \\
\hline Past smoker & $513(27.65)$ & $2(0.73)$ & \\
\hline Current smoker & $202(10.89)$ & $27(9.87)$ & \\
\hline Alcohol consumption & & & 0.001 \\
\hline Nondrinker & $1,152(62.10)$ & $196(72.06)$ & \\
\hline Drinker & $703(37.90)$ & 76 (27.94) & \\
\hline Educational level & & & $<0.001$ \\
\hline Lack of schooling/unschooled study & $188(10.12)$ & $56(20.44)$ & \\
\hline Elementary school & $708(38.13)$ & $146(53.28)$ & \\
\hline Junior high/high school & $715(38.50)$ & $71(25.91)$ & \\
\hline$\geq$ College & $246(13.25)$ & $1(0.36)$ & \\
\hline Household income (1,000 won/mo) & & & $<0.001$ \\
\hline$<400$ & $167(8.96)$ & $68(31.05)$ & \\
\hline $400-900$ & $401(21.51)$ & $92(42.01)$ & \\
\hline$\geq 900$ & $1,296(69.53)$ & $59(26.94)$ & \\
\hline Experience of being basic living recipient & & & 0.188 \\
\hline No & $1,681(89.80)$ & $253(92.34)$ & \\
\hline Yes & $191(10.20)$ & $21(7.66)$ & \\
\hline No. of family members living together & & & $<0.001$ \\
\hline 0 & $373(19.91)$ & $77(27.80)$ & \\
\hline 1 & $932(49.76)$ & $173(62.45)$ & \\
\hline 2 & $568(30.33)$ & $27(9.75)$ & \\
\hline
\end{tabular}

Values are presented as number (\%) for categorical variables or mean \pm standard deviation for continuous variables. "By $\chi^{2}$-test for categorical variables and Student $t$-test for continuous variables. 
Table 2. Unadjusted prevalence, awareness, and control of hypertension and diabetes according to area of residence

\begin{tabular}{cccr}
\hline & \multicolumn{2}{c}{ Area of residence } & \\
\cline { 2 - 3 } Variable & $\begin{array}{c}\text { General population } \\
(\mathrm{n}=1,873)\end{array}$ & $\begin{array}{c}\text { Primary care underserved } \\
\text { area }(\mathrm{n}=279)\end{array}$ & \\
\hline Hypertension & & & \\
Prevalence & $1,138(60.79)$ & $1,97(70.61)$ & 0.002 \\
Awareness & $964(84.71)$ & $143(72.59)$ & $<0.001$ \\
Control & $695(61.07)$ & $61(30.96)$ & $<0.001$ \\
Diabetes & & & \\
Prevalence & $491(26.23)$ & $66(23.66)$ & 0.360 \\
Awareness & $407(82.89)$ & $58(87.88)$ & 0.306 \\
Control & $232(47.25)$ & $42(63.64)$ & 0.012 \\
\hline
\end{tabular}

Values are presented as number (\%).

"By $\chi^{2}$-test for categorical variables.

Table 3. Adjusted odds ratio for the prevalence, awareness, treatment, and control of hypertension and diabetes according to area of residence

\begin{tabular}{|c|c|c|c|}
\hline \multirow[b]{2}{*}{ Variable } & \multicolumn{2}{|c|}{ Area of residence } & \multirow[b]{2}{*}{ P-value* } \\
\hline & $\begin{array}{l}\text { General population } \\
\qquad(\mathrm{n}=1,873)\end{array}$ & $\begin{array}{c}\text { Primary care underserved } \\
\text { area }(n=279)\end{array}$ & \\
\hline \multicolumn{4}{|l|}{ Hypertension } \\
\hline Prevalence & 1 & $1.17(0.83-1.67)$ & 0.374 \\
\hline Awareness & 1 & $0.40(0.25-0.64)$ & $<0.001$ \\
\hline Control & 1 & $0.27(0.18-0.41)$ & $<0.001$ \\
\hline \multicolumn{4}{|l|}{ Diabetes } \\
\hline Prevalence & 1 & $0.88(0.61-1.26)$ & 0.479 \\
\hline Awareness & 1 & $1.06(0.43-2.59)$ & 0.899 \\
\hline Control & 1 & $1.67(0.87-3.19)$ & 0.123 \\
\hline
\end{tabular}

Values are presented as odds ratios (95\% confidence intervals). Multivariate adjusted for age, sex, body mass index, smoking/alcohol status, educational level, income, and the number of family members living together.

"By $\chi^{2}$-test for categorical variables.

We calculated the multivariate OR for prevalence, awareness, and control of hypertension and diabetes according to area of residence by adjusting for age, sex, BMI, smoking/alcohol status, educational level, income, and number of family members living together (Table 3 ). The aOR (95\% CIs) of the prevalence of hypertension, after adjustment, showed no statistically significant difference for the area of residence (aOR, 1.17; 95\% CI, 0.83-1.67; $\mathrm{P}=0.374$ ). Among hypertensive participants, PCUA residents had a significantly higher risk of underdiagnosed and uncontrolled hypertension compared to the general population (aOR, 0.40; 95\% CI, 0.25-0.64; $\mathrm{P}<0.001$; aOR, 0.27; 95\% CI, 0.18$0.41 ; \mathrm{P}<0.001$, respectively). There were no significant associations between prevalence, awareness, and control of diabetes and area of residence after adjustments (for all $\mathrm{P}>0.05$ ).

\section{Subjective Health Status, Health-Related Quality of Life, and Area of Residence}

Table 4 shows the SHS and EQ-5D results based on area of residence before adjustment. The residents of PCUAs reported more problems with all QoL dimensions compared to the general population (for all
Table 4. Health-related quality of life and subjective health status of the participants according to area of residence in the unadjusted model

\begin{tabular}{|c|c|c|c|}
\hline \multirow[b]{2}{*}{ Variable } & \multicolumn{2}{|c|}{ Area of residence } & \multirow[b]{2}{*}{ P-value } \\
\hline & $\begin{array}{l}\text { General population } \\
\qquad(\mathrm{n}=1,873)\end{array}$ & $\begin{array}{l}\text { Primary care underserved } \\
\text { area }(n=279)\end{array}$ & \\
\hline Mobility & & & $<0.001$ \\
\hline 1 & $1,261(67.36)$ & 87 (31.18) & \\
\hline 2 & 611 (32.64) & 192 (68.82) & \\
\hline Self-care & & & $<0.001$ \\
\hline 1 & 1,695 (90.54) & 222 (79.57) & \\
\hline 2 & $177(9.46)$ & 57 (20.43) & \\
\hline Usual activities & & & $<0.001$ \\
\hline 1 & 1,524 (81.41) & 88 (31.54) & \\
\hline 2 & 348 (18.59) & $191(68.46)$ & \\
\hline Pain/discomfort & & & $<0.001$ \\
\hline 1 & 1,237 (66.08) & 60 (21.51) & \\
\hline 2 & 635 (33.92) & 219 (78.49) & \\
\hline Anxiety/depression & & & $<0.001$ \\
\hline 1 & 1,597 (85.31) & $150(53.76)$ & \\
\hline 2 & 275 (14.69) & $129(46.24)$ & \\
\hline $\begin{array}{l}\text { Subjective health } \\
\text { status }^{\dagger}\end{array}$ & & & $<0.001$ \\
\hline 1 & $1,316(70.30)$ & $104(37.28)$ & \\
\hline 2 & $556(29.7)$ & 175 (62.72) & \\
\hline EQ-5D index & $0.87 \pm 0.003$ & $0.73 \pm 0.011$ & $<0.001$ \\
\hline
\end{tabular}

Values are presented as number (\%) for categorical variables or mean \pm standard error for EQ-5D index. Mobility, self-care, usual activities, pain/discomfort, anxiety/ depression: 1, no problem; 2, yes problem (some or severe problems).

EQ-5D index, EuroQol-5D index.

'By $\chi^{2}$-test for categorical variables and Student t-test for continuous variables. ${ }^{\dagger} 1$, good; 2 , bad.

Table 5. Adjusted odds ratio for health-related quality of life and subjective health status of the participants according to area of residence

\begin{tabular}{|c|c|c|c|}
\hline \multirow[b]{2}{*}{ Variable } & \multicolumn{2}{|c|}{ Area of residence } & \multirow[b]{2}{*}{ P-value } \\
\hline & $\begin{array}{l}\text { General population } \\
\qquad(\mathrm{n}=1,873)\end{array}$ & $\begin{array}{c}\text { Primary care underserved } \\
\text { area }(n=279)\end{array}$ & \\
\hline $\begin{array}{l}\text { Poor subjective health } \\
\text { status }\end{array}$ & 1.00 & $3.08(2.19-4.32)$ & $<0.001$ \\
\hline \multicolumn{4}{|l|}{ Problem in EQ-5D } \\
\hline Mobility & 1.00 & $3.15(2.21-4.50)$ & $<0.001$ \\
\hline Self-care & 1.00 & $1.19(0.76-1.86)$ & 0.448 \\
\hline Usual activities & 1.00 & 7.15 (4.94-10.38) & $<0.001$ \\
\hline Pain/discomfort & 1.00 & $6.31(4.30-9.25)$ & $<0.001$ \\
\hline Anxiety/depression & 1.00 & $4.18(2.95-5.93)$ & $<0.001$ \\
\hline$E Q-5 D$ index & 1.00 & $0.91(0.89-0.92)$ & $<0.001$ \\
\hline
\end{tabular}

Values are presented as odds ratios (95\% confidence intervals). Multivariate adjusted for age, sex, body mass index, smoking/alcohol status, educational level, income, and the number of family members living together.

EQ-5D, EuroQol-5D.

"By $\chi^{2}$-test for categorical variables and Student t-test for continuous variables.

$\mathrm{P}<0.001)$. Participants from PCUAs had a lower EQ-5D index $(\mathrm{P}<0.001)$ and poorer SHS $(\mathrm{P}<0.001)$ than the participants from the general population.

The aOR values for poor HRQoL were calculated by adjusting for age, sex, BMI, smoking/alcohol status, educational level, income, and 
number of family members living together (Table 5). After adjustment, residents of PCUAs had poorer SHS (aOR, 3.08; 95\% CI, 2.19-4.32) compared to the general population. They also exhibited significantly higher OR for problems in all HRQoL dimensions, with the exception of self-care, compared to the general population. Regarding the mobility QoL dimension, the OR of those in PCUAs was 3.15 (95\% CI, 2.214.50; $\mathrm{P}<0.001)$. For usual activities, the $\mathrm{OR}$ was the highest magnitude of 7.15 (95\% CI, 4.94-10.38; $\mathrm{P}<0.001$ ). Regarding pain/discomfort, the OR was 6.31 (95\% CI, 4.30-9.25; $\mathrm{P}<0.001)$. For anxiety/depression, the OR was 4.18 (95\% CI, 2.95-5.93; $\mathrm{P}<0.001$ ). For poor SHS, the OR was 3.08 (95\% CI, 2.19-4.32; $\mathrm{P}<0.001$ ). The OR for EQ-5D index was significantly lower in PCUA residents (OR, 0.91; 95\% CI, 0.89-0.92; $\mathrm{P}<0.001$ ) than that in the general population.

\section{DISCUSSION}

Residents of PCUAs were significantly underdiagnosed and undercontrolled in hypertension, but not in diabetes. They were more likely to feel unhealthy and had poorer HRQoL compared to the general population.

The impact of urban residence varies in several studies. Several studies have revealed the significant association between urban residence and prevalence of hypertension and diabetes in Korea., ${ }^{911,12,21)}$ Other studies have reported no significant differences in prevalence, awareness, or control of hypertension and diabetes between rural and urban areas. ${ }^{10)}$ Health disparities have been attributed to disparities in access and quality of health care. ${ }^{22)}$ In the present study, the PCUAs were not only at the "eup" and "myun" levels, which represent the smallest and most rural regional unit in Korea. Namwon city, a small city, had insufficient primary care. Residents of this city also showed poor control of chronic disease and poorer QoL compared to the general population. We suggest that insufficient primary care, rather than rurality, can contribute to poorer control of chronic diseases and QoL.

Ogedegbe $^{22)}$ described that the barriers to optimal hypertension control could be (1) patient-related, (2) physician-related, and (3) medical environment-/health care system-related. In the current study, the residents of the underserved areas were older, had lower educational levels and incomes, and consisted of fewer smokers and drinkers compared to the general population. Lack of awareness and inadequate control of hypertension remained higher in the underserved areas after adjustments for patient-related factors. Although physician-related factors vary, the present study strongly suggests that insufficient primary care was a strong factor influencing the awareness and control of hypertension.

Studies have examined the association between area of residence and HRQoL in Korea. HRQoL tends to be lower for residents living around industrial complexes, specifically for those living around power plants, than those not living around industrial complexes. ${ }^{3)}$ Rural residents are more likely to believe they are unhealthy than do urban residents. ${ }^{23,24)}$ In the current study, all EQ-5D indicators, except for selfcare, EQ-5D index, and SHS were lower in the underserved areas after adjusting for confounding factors, such as SES and healthy behavior, than that in the general population. These residents felt "unhealthier" and had limited ability to exercise and perform daily activities. Furthermore, they felt pain/discomfort more frequently and more anxiety/depression than the general population. These results support our hypothesis that primary care affects the subjective health of residents and their QoL.

This study has several limitations. First, as a cross-sectional study, the interpretation of the causes and results is limited. Second, we could not check whether or not the participants took hypertensive or diabetic medications; therefore, current medication was not included in the definition of hypertension and diabetes in both the general population and residents of underserved areas, possibly underestimating the prevalence. Moreover, we did not include serum hemoglobin Alc (HbAlc) level in the definition and control criteria of diabetes; this limitation could also have resulted in underestimated prevalence. Other studies have either included ${ }^{1,9,13)}$ or excluded ${ }^{7,8,11,25)} \mathrm{HbAlc}$ level as a criterion of diabetes management. Third, the study population was smaller ( $n=279)$ compared to the control group. Thus, we are unable to exclude the possibility of a selection bias. Our group could not represent all 315 PCUAs.

Despite these limitations, this study is significant because this is the first to investigate health status according to accessibility of primary care in Korea. To fully determine the health status in underserved areas, further studies including larger and randomized populations are required.

In conclusion, the PCUA residents in this study were underdiagnosed and under-controlled for hypertension. However, there was no difference in the management of diabetes between the two groups. Additionally, the PCUA residents reported a poorer subjective health and HRQoL compared to the general population. Our study suggests that primary care contributes to awareness and control of chronic diseases, subjective health, and QoL in communities.

\section{CONFLICT OF INTEREST}

No potential conflict of interest relevant to this article was reported.

\section{ACKNOWLEDGMENTS}

We are grateful to the mobile team of the Public Health and Medical Service Department of Seoul National University Hospital.

\section{ORCID}

Min So Kim: https://orcid.org/0000-0001-8800-4425

Kyae Hyung Kim: https://orcid.org/0000-0001-9954-6422

Sang Min Park: https://orcid.org/0000-0002-7498-4829

Jong-goo Lee: https://orcid.org/0000-0003-4833-1178 


\section{REFERENCES}

1. Lee HY, Hahm MI, Choi KS, Jun JK, Suh M, Nam CM, et al. Different socioeconomic inequalities exist in terms of the prevention, diagnosis and control of diabetes. Eur J Public Health 2015;25:961-5.

2. Fine JT, Colditz GA, Coakley EH, Moseley G, Manson JE, Willett WC, et al. A prospective study of weight change and health-related quality of life in women. JAMA 1999;282:2136-42.

3. Kim H, Roh S. Relationship between residential district and health-related quality of life in Chungnam industrial complex area. Environ Health Toxicol 2016;31:e2016017.

4. Yoon TH. Regional health inequalities in Korea: the status and policy tasks. J Crit Soc Policy 2010;30:49-77.

5. Cho HJ. Equity in health care: current situation in South Korea. J Korean Med Assoc 2013;56:184-94.

6. Jeong BG, Jung KY, Kim JY, Moon OR, Lee YH, Hong YS, et al. The relationship between regional material deprivation and the standardized mortality ratio of the community residents aged 15-64 in Korea. J Prev Med Public Health 2006;39:46-52.

7. Kim YJ, Jeon JY, Han SJ, Kim HJ, Lee KW, Kim DJ. Effect of socio-economic status on the prevalence of diabetes. Yonsei Med J 2015;56:6417.

8. Min H, Chang J, Balkrishnan R. Sociodemographic risk factors of diabetes and hypertension prevalence in republic of Korea. Int J Hypertens 2010;2010:410794.

9. Jeon YJ, Kim CR, Park JS, Choi KH, Kang MJ, Park SG, et al. Health inequalities in hypertension and diabetes management among the poor in urban areas: a population survey analysis in south Korea. BMC Public Health 2016;16:492.

10. Zhang Y, Zhou Z, Gao J, Wang D, Zhang Q, Zhou Z, et al. Health-related quality of life and its influencing factors for patients with hypertension: evidence from the urban and rural areas of Shaanxi Province, China. BMC Health Serv Res 2016;16:277.

11. Lee HY, Won JC, Kang YJ, Yoon SH, Choi EO, Bae JY, et al. Type 2 diabetes in urban and rural districts in Korea: factors associated with prevalence difference. J Korean Med Sci 2010;25:1777-83.

12. Lee HM, Kim YM, Lee CH, Shin JH, Kim MK, Choi BY. Awareness, treatment and control of hypertension and related factors in the jurisdictional areas of primary health care posts in a rural community of Korea. J Prev Med Public Health 2011;44:74-83.

13. Kim HY, Yun WJ, Shin MH, Kweon SS, Ahn HR, Choi SW, et al. Management of diabetic mellitus in low-income rural patients. J Prev Med Public Health 2009;42:315-22.

14. Ministry of Health and Welfare. Monitoring of medically underserved area. Sejong: Ministry of Health and Welfare; 2014.

15. Na BJ, Lee JY, Kim HJ. Are public health physicians still needed in medically underserved rural areas in Korea? Medicine (Baltimore) 2017;96:e6928.

16. Kee SI. Validity and reliability evaluation for EQ-5D in Korea. Cheongju: Korea Center for Disease Control and Prevention; 2011.

17. Byun JS. Sampling design of the 6th KNHANES and weighting adjustment of the 5th KNHANES. Cheongju: Korea Centers for Disease Control and Prevention; 2012.

18. Kim M, Chung W, Lim S, Yoon S, Lee J, Kim E, et al. Socioeconomic inequity in self-rated health status and contribution of health behavioral factors in Korea. J Prev Med Public Health 2010;43:50-61.

19. Kwak Y, Kim Y. Quality of life and subjective health status according to handgrip strength in the elderly: a cross-sectional study. Aging Ment Health 2019;23:107-12.

20. Lee YK, Nam HS, Chuang LH, Kim KY, Yang HK, Kwon IS, et al. South Korean time trade-off values for EQ-5D health states: modeling with observed values for 101 health states. Value Health 2009;12:1187-93.

21. Lee MS. Health inequalities among Korean adults: socioeconomic status and residential area differences. Korean J Sociol 2005;39:183209.

22. Ogedegbe G. Barriers to optimal hypertension control. J Clin Hypertens (Greenwich) 2008;10:644-6.

23. Lee JA, Park JH, Kim M. Social and physical environments and selfrated health in urban and rural communities in Korea. Int J Environ Res Public Health 2015;12:14329-41.

24. Choi BM, Lee JY. Comparison of life satisfaction between urban and rural residents. Korean Soc Sci J 2008;35:31-54.

25. Hwang J, Shon C. Relationship between socioeconomic status and type 2 diabetes: results from Korea National Health and Nutrition Examination Survey (KNHANES) 2010-2012. BMJ Open 2014;4:e005710. 